\title{
Ubiquitin-Specific Peptidase 22 Contributes to Colorectal Cancer Stemness and Chemoresistance via Wnt/ $\beta$-Catenin Pathway
}

\author{
Shixiong Jiang Chenxin Song Xinyu Gu Muhong Wang Dazhuang Miao \\ Jiachen Lv Yanlong Liu \\ Department of Colorectal Surgery, Harbin Medical University Cancer Hospital, Harbin, Heilongjiang, \\ P.R. China
}

\section{Key Words}

Usp22 • Colorectal cancer $\cdot$ Wnt/ß-catenin pathway • Stemness $・$ Chemoresistance

\begin{abstract}
Background/Aims: Two major barriers to the successful treatment of colorectal cancer (CRC) are the development of stem cell-like characteristics (stemness) and chemoresistance. Ubiquitin-specific peptidase 22 (USP22) is a deubiquitinating enzyme and putative CRC marker that has emerged as a potential cause of both phenomena in CRC. There is evidence that USP22 acts through the Wnt/ $\beta$-catenin pathway and that downregulation of the latter may reduce chemoresistance. Methods: In this study, we used CRC tissue specimens from human patients as well as human CRC cell lines to evaluate the role of USP22 in CRC stemness and chemoresistance in vitro and in vivo. RT-PCR and western blot were used for gene expression analyses. Immunohistochemistry was performed for USP22 expression in clinical samples. CD133 levels were analyzed by flow cytometry. Sphere formation and MTT assays were used for self-renewal and proliferation analysis. Chemoresistance was evaluated by cell viability and sphere formation assays. Results: We found a significant increase of USP22 in recurrent CRC and chemoresistant CRC cells as compared to primary CRC and non-chemoresistant CRC cells, respectively. We then demonstrated that USP22 mediates CRC cell chemoresistance through the Wnt/ $\beta$-catenin pathway and that reducing USP22 in CRC cells diminishes chemoresistance. Conclusions: Having established the crucial role of USP22 in CRC stemness and chemoresistance, this study suggests that USP22 may be an ideal genetic target in the treatment of chemoresistant CRC.




\section{Cellular Physiology Cell Physiol Biochem 2018;46:1412-1422 \begin{tabular}{l|l} 
and Biochemistry Published onlıne: April23, 2018 & $\begin{array}{l}\text { (c) } 2018 \text { The Author(s). Published by S. Karger AG, Basel } \\
\text { www.karger.com/cpb }\end{array}$
\end{tabular} \\ Jiang et al.: USP22 Contributes to Colorectal Cancer Stemness and Chemoresistance}

\section{Introduction}

Colorectal cancer (CRC) is the third most common cancer and the second leading cause of cancer-related death in Western countries [1]. The 5-year relative survival rate of patients with metastasized CRC is only 8\% [2]. A strong prognostic determinant for CRC patients is the success or failure of their chemotherapy regimen. Because of this, chemoresistance poses a great threat to successful treatment. Even though almost $50 \%$ of patients respond to systemic therapies, nearly all patients develop chemoresistance [1]. Therefore, understanding mechanisms of chemoresistance promises to prevent or reverse chemoresistance and thus revolutionize CRC treatment with chemotherapy. In this study, we focus on CRC chemoresistance to 5-fluorouracil (5-FU), which, despite the advent of targeted molecular therapies, is a systemic therapy that remains central to the treatment of CRC [3].

While the mechanism of CRC chemoresistance is unknown and likely varies by cancer genotype and phenotype, there is strong evidence to support the idea that it is a tumor's cancer stem cells (CSCs) that cause chemoresistance [4]. CSCs promote tumor growth, invasion, and metastasis--all of which negatively impact prognosis. Furthermore, because CSCs cycle slowly, they increase chemoresistance and thus tumor recurrence [2]. Reducing a tumor's stemness may therefore also reduce its chemoresistance [5]. Thus, in an investigation of chemoresistance, it is also important to consider stemness, or the ability of a cell to selfrenew and differentiate into various cell types [6].

Ubiquitin-specific protease 22 (USP22) is a gene that may be crucial to the stemness of CRC. It has been shown to promote cell cycle progression and tumorigenesis [7-9]. Furthermore, USP22 expression correlates with CRC progression and therapy failure [10]. In this study, we analyzed the levels of USP22 expression in human primary and recurrent CRC tissues, CRC cell lines, CRC cells with CD133 surface antigen (a colon CSC marker), and 5-FU resistant CRC cells, to determine the impacts of USP22 expression on CRC recurrence, stemness, and chemoresistance [2]. We also assessed the relationship between USP22 and the Wnt/ $\beta$-catenin signaling pathway, which has been shown to contribute to stemness, tumorigenesis, and chemoresistance $[11,12]$.

The Wnt/ $\beta$-catenin pathway controls cell proliferation and stem cell self-renewal [11]. Aberrant and excessive Wnt signaling contribute to CRC growth as well as to CSC maintenance $[13,14]$. Increased Wnt signaling indicates a poor prognosis for patients with CRC, and disrupting Wht pathway activation prevents CRC progression [15]. Given the evidence for USP22's promotion of $\beta$-catenin nuclear localization, which is necessary for Wnt pathway activation, we hypothesized that USP22 maintains CRC cell stemness and tumorigenesis through Wnt/ $\beta$-catenin signaling [7]. USP22 knockdown in CRC cell lines does in fact reduce $W n t / \beta$-catenin signaling, suggesting a greater role for USP22 in CRC progression than was previously supposed. Overall, the present study demonstrates that USP22 mediates CRC cell stemness, tumorigenesis, and chemoresistance through the Wnt/ $\beta$-catenin signaling pathway.

\section{Materials and Methods}

\section{Tissue specimens}

This study was approved by the Research Ethics Committee of the Affiliated Tumor Hospital of Harbin Medical University (Harbin, China). All patients provided written informed consent. Paired fresh primary and recurrent colorectal cancer (CRC) tissues were obtained from 4 patients who underwent surgery at the Affiliated Tumor Hospital of Harbin Medical University. The specimens were snap frozen in liquid nitrogen and stored at $-80^{\circ} \mathrm{C}$ until processing.

\section{Cell culture}

Human CRC cell lines (Caco2, HT29, HCT15, HCT116, SW620 and SW480) were obtained from the Shanghai Institutes for Biological Sciences of the Chinese Academy of Sciences (ATCC). Cells were cultured at $37^{\circ} \mathrm{C}$ in $5 \% \mathrm{CO}_{2}$ atmosphere in RPMI-1640 medium (Hyclone, Logan, UT), supplemented with $10 \%$ 


\section{Cellular Physiology Cell Physiol Biochem 2018;46:1412-1422

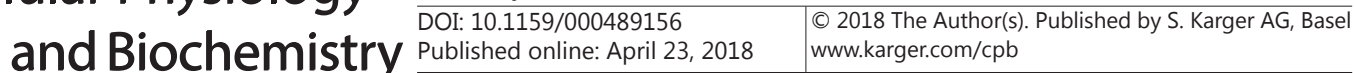 \\ Jiang et al.: USP22 Contributes to Colorectal Cancer Stemness and Chemoresistance}

bovine calf serum (Hyclone) and $2 \mathrm{mM}$ L-glutamine. Caco2 and HCT15 CD133+ cells were cultured in RPMI-1640 medium, supplemented with B27, heparin, N2 supplement, $20 \mathrm{ng} / \mathrm{ml} \mathrm{EGF} \mathrm{and} 20 \mathrm{ng} / \mathrm{ml}$ bFGF.

Reverse transcription PCR (RT-PCR)

Total RNA was isolated from CRC tissues and cell lines using Trizol reagent (Invitrogen, Carlsbad, CA) and reverse transcribed into cDNA using Superscript First-Strand Synthesis System (Invitrogen) according to the manufacturer's instructions. The primers used are listed in Table 1. PCR conditions were: initial denaturation at $95^{\circ} \mathrm{C}$ for $2 \mathrm{~min}$, followed by 30 cycles of amplification at $95^{\circ} \mathrm{C}$ for 30 $\mathrm{s}, 55^{\circ} \mathrm{C}$ for $45 \mathrm{~s}$, and $72^{\circ} \mathrm{C}$ for $1 \mathrm{~min}$, and a final extension at $72^{\circ} \mathrm{C}$ for $15 \mathrm{~min}$.

\section{Western blot}

Total protein from CRC tissues and cell lines was extracted in a lysis buffer consisting of $20 \mathrm{mM}$ Tris- $\mathrm{HCl}(\mathrm{pH} 7.5)$, 2 mM EDTA, $150 \mathrm{mM} \mathrm{NaCl}, 1 \%$ Triton $\mathrm{X}-100$, and protease inhibitors. Protein was analyzed in the supernatant by the Bradford method (BioRad, Hercules). Proteins in all samples were separated by SDS-PAGE (10\%) and transferred onto nitrocellulose membrane. Membranes were probed with primary antibodies overnight at $4^{\circ} \mathrm{C}$. After washing, the membranes were incubated with the HRP-conjugated secondary antibody for $1 \mathrm{~h}$. The following antibodies were used: antibodies against USP2 2 and $\beta$-catenin were from Abcam (Cambridge, MA); antibodies against MYC, Sox2 and Axin2 were from Cell Signaling Technology (Danvers, MA); antibodies against CD133, CD44, Cyclin D1 and GSK3 $\beta$ were from Santa Cruz Biotechnology (Santa Cruz, CA).

\section{USP22 siRNA Sequences and Transfection}

The USP22 sequence-specific siRNA and scramble control siRNA were designed and synthesized by Invitrogen. The sequences of siRNA were as follows: USP22 siRNA, 5'-TGCTGTCAAGCTCCCGTTTGGTTGGTGTTTTGGCCACTGACTGACACCAACCACGGGAGCTTGA-3' (forward) and 5'-CCTGTCAAGCTCCCGTGGTTGGTGTCAGTCAGTGGCCAAAACACCAACCAAACGGGAGCTTGAC-3' (reverse); Scramble control siRNA, 5'-TGCTGAAATGTACTGCGCGTGGAGACGTTTTGGCCACTGACTGACGTCTCCACGCAGTACATTT-3' (forward) and 5'-CCTGAAATGTACTGCGTGGAGACGTCAGTCAGTGGCCAAAACGTCTCCACGCGCAGTACATTTC-3' (reverse). FuGENE ${ }^{\circledR}$ HD Transfection Reagent (Invitrogen) was used for cell transfection, following the manufacturer's instructions. 48 hours after transfection, cells were collected for next steps.

Immunohistochemistry

Paraffin-embedded tissue blocks from four patients were retrieved from the Pathology Department, and $5 \mu \mathrm{m}$ thick sections were prepared for standard immunohistochemistry using EnVisionTM's immunohistochemistry methods. Briefly, the tissue sections were deparaffinized in xylene and rehydrated

Table 1. Primer sequences for RT-PCR

\begin{tabular}{|c|c|}
\hline Primers & sequences \\
\hline USP22 forward & 5'-GAAGGTTGGCATGGAAGA-3' \\
\hline USP22 reverse & 5'-TGTAATTGGATTCAGGTGGA-3' \\
\hline CD133 forward & 5'-GGGAGAACAATAATAGGATATTTTGAA-3' \\
\hline CD133 reverse & 5'-CGATGCCACTTTCTCACTGAT-3' \\
\hline CD44 forward & 5'-TTTGCATTGCAGTCAACAGTC-3' \\
\hline CD44 reverse & 5'-GTTACACCCCAATCTTCATGTCCAC-3' \\
\hline Sox2 forward & 5'-ACACCAАТСССАТССАСАСТ-3' \\
\hline Sox2 reverse & 5'-GCAAACTTCCTGCAAAGCTC-3' \\
\hline Axin2 forward & 5'-GGTGTTTGAGGAGATCTGGG-3' \\
\hline Axin2 reverse & 5'-TGCTCACAGCCAAGACAGTT-3' \\
\hline MYC forward & 5'-GCCACGTCTCCACACATCAG-3' \\
\hline MYC reverse & 5'-TCTTGGCAGCAGGATAGTCCTT-3' \\
\hline Cyclin D1 forward & 5'-ACCTGGATGCTGGAGGTCT-3' \\
\hline Cyclin D1 reverse & 5'-GCTCCATTTGCAGCAGCTC-3' \\
\hline Wnt1 forward & 5'-CCACCTCTTCGGCAAGATCGTCAA-3' \\
\hline Wnt1 reverse & 5'-GTGGCATTTGCACTCTTGGCGCAT-3' \\
\hline Wnt2b forward & 5'-CCGAGGGTATGACACAACTC-3' \\
\hline Wnt $2 \mathrm{~b}$ reverse & 5'-GTGGAGGGAAGAATGAGGTT-3' \\
\hline Wnt3a forward & 5'-CTCCTCTCGGATACCTCTTAGTG-3' \\
\hline Wnt3a reverse & 5'-GCATGATCTCCACGTAGTTCCTG-3' \\
\hline LRP5 forward & 5'-GACCCAGCCCTTTGTTTTGAC-3' \\
\hline LRP5 reverse & 5'-TGTGGACGTTGATGGTATTGGT-3' \\
\hline LRP6 forward & 5'-CCCATGCACCTGGTTCTACT-3' \\
\hline LRP6 reverse & 5'-CCAAGCCACAGGGATACAGT-3' \\
\hline Axin forward & 5'-СССТСССАССТСТTСАТСС-3' \\
\hline Axin reverse & 5'-CAGTCAAACTCGTCGCTCAC-3' \\
\hline GSK3 $\beta$ forward & 5'-CAGCAAGGTGACAACAGTGG-3' \\
\hline GSK3 $\beta$ reverse & 5'-GGAACATAGTCCAGCACCAGA-3' \\
\hline APC forward & 5'-TGTCCCTCCGTTCTTATGGAA-3' \\
\hline APC reverse & 5'-TCTTGGAAATGAACCCATAGGAA-3' \\
\hline GAPDH forward & 5'-GCCAAAAGGGTCATCATCTC-3' \\
\hline GAPDH reverse & 5'-GTAGAGGCAGGGATGATGTTC-3' \\
\hline
\end{tabular}




\section{Cellular Physiology Cell Physiol Biochem 2018;46:1412-1422

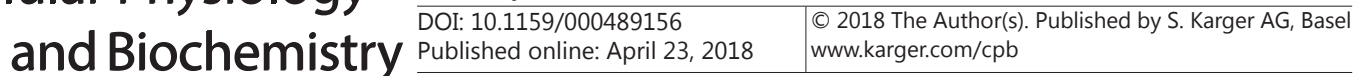 \\ Jiang et al.: USP22 Contributes to Colorectal Cancer Stemness and Chemoresistance}

through an ethanol gradient. Samples were blocked in $20 \%$ normal serum and incubated overnight at $4{ }^{\circ} \mathrm{C}$ with a primary antibody against USP22 (Abcam) at a dilution of 1:100. The sections were washed with phosphate-buffered saline (PBS) and then incubated with secondary antibody at room temperature for 1 h. The color reaction was processed with 3, 3'-diaminobenzidine (DAB) solution and then viewed under a microscope. For fluorescent staining, the slides were incubated with primary anti-USP22 antibody overnight after blocking and then incubated with AlexaFluor 488-conjugated secondary antibody for $1 \mathrm{~h}$. Nuclear counterstaining was assessed by incubating slides with 4, 6-diamidino-2-phenylindole (DAPI). Slides were observed under a fluorescence microscope equipped with a digital camera (Nikon, japan).

\section{CD133+ cell isolation}

The CD133+ CRC cells were isolated from Caco2 and HCT15 cell lines using magnetic-activated cell sorting (MACS; Miltenyi, Bergisch Gladbach, Germany), according to the manufacturer's instructions. Briefly, CRC cells were collected and centrifuged for $5 \mathrm{~min}$. The supernatant was removed and 20 $\mu \mathrm{L}$ CD133 microbeads were mixed in and incubated for 15 minutes at $4^{\circ} \mathrm{C}$. The cells were washed twice to remove the uncombined microbeads. The CD133+ cells were isolated by a magnetic separation column. In order to verify the efficiency of cell isolation, the isolated cells were stained with CD133-PE and analyzed by flow cytometry (BD Biosciences, San Jose, CA, USA).

\section{Sphere formation assays}

CRC cells $\left(1 \times 10^{3}\right.$ cells/well) were plated in 6-well plates with ultra-low adherence (Corning, Corning, NY) and cultured in RPMI-1640 medium, supplemented with B27, heparin, N2 supplement, 20 ng/ml EGF and $20 \mathrm{ng} / \mathrm{ml} \mathrm{bFGF}$ for three days to form spheres.

\section{MTT assays}

Cell viability was assayed using the CellTiter 96® AQueous One Solution Cell Proliferation Assay (CellTiter96; Promega) according to the manufacturer's instructions. Briefly, the cells were seeded onto 96-well plates and cultured for up to 7 days. At the end of each period, $10 \mu \mathrm{L}$ MTT solution was added and the cells were incubated for an additional $4 \mathrm{~h}$, after which $150 \mu \mathrm{L}$ dimethyl sulfoxide (DMSO) was added to each well and mixed thoroughly. The optical density of each well was measured with a spectrophotometer (UV5100, Shanghai).

\section{5-FU resistant cell establishment}

5-FU resistant CRC cells were generated by continuous exposure to increasing concentrations of 5-FU (from 5 to $30 \mu \mathrm{g} / \mathrm{ml}$ ) with repeated subculture until fully resistant to 5-FU. Cells were first cultured in growth medium with $5 \mu \mathrm{g} / \mathrm{ml} \mathrm{5-FU} \mathrm{for} \mathrm{two} \mathrm{months,} \mathrm{and} \mathrm{the} \mathrm{concentration} \mathrm{of} 5$-FU increased $5 \mu \mathrm{g} / \mathrm{ml}$ every two months.

\section{Animal model}

Animal protocols were approved by the Institutional Animal Care and Use Committee (IACUC) of the Affiliated Tumor Hospital of Harbin Medical University. Caco2 cells with stable knockdown of USP22 were set up by USP22 shRNA transfection. 40 nude mice (4-6 weeks old) were maintained in a pathogenfree environment at the experimental animal center of the Affiliated Tumor Hospital of Harbin Medical University, and randomly divided into eight groups: four Control groups, injected subcutaneously with control Caco 2 cells $\left(10 \times 10^{8}, 1 \times 10^{8}, 0.1 \times 10^{8}\right.$ and $0.01 \times 10^{8}$, respectively); and four USP22 knockdown groups, injected subcutaneously with USP22 stable knockdown Caco2 cells $\left(10 \times 10^{8}, 1 \times 10^{8}, 0.1 \times 10^{8}\right.$ and $0.01 \times 10^{8}$, respectively). After $60 \mathrm{~d}$ of injection, the ratios of mice without tumor growths were calculated for each group.

\section{Statistical analysis}

Statistical analysis was performed using GraphPad software (version 5.0). The differences between paired groups were analyzed by Student's $t$-test. Data for multiple groups were analyzed by one-way ANOVA. $P$ values less than 0.05 were considered statistically significant. The data are expressed as mean \pm standard error of the mean (SEM). 


\section{Cellular Physiology Cell Physiol Biochem 2018;46:1412-1422 \begin{tabular}{l|l} 
and Biochemistry & DOI: 10.1159/000489156 \\
Publisher 2018 The Author(s). Published by S. Karger AG, Basel \\
www.karger.com/cpb
\end{tabular}

\section{Results}

USP22 expression is significantly increased in recurrent colorectal cancer tissues

In order to investigate USP22 function in colorectal cancer (CRC) recurrence, we first analyzed USP22 mRNA expression in paired primary and recurrent CRC tissues from four CRC patients. We found that USP22 mRNA is significantly increased in recurrent tissues compared to primary tissues (Fig. 1A). Western blot showed overexpression of USP22 protein in recurrent tissues (Fig. 1B). Immunohistochemistry staining further confirmed USP22 upregulation in recurrent CRC (Fig. 1C). We performed western blot for USP22 expression in six CRC cell lines (Caco2, HT29, HCT15, HCT116, SW620 and SW480) and found that USP22 was increased in all cell lines compared with primary colorectal cancer tissues (Fig. 1D). Overall, these results demonstrate that USP22 is significantly and consistently upregulated in recurrent CRC tissues and in CRC cell lines.

USP22 is required for CRC stemness and tumorigenesis

To evaluate the effects of USP22 on CRC cell stemness and tumorigenesis, we isolated CD133+ Caco2 and HCT15 cells using the MicroBeads method. Positive CD133 expression was confirmed with flow cytometry analysis (Fig. 2A). We then analyzed USP22 expression in CD133- and CD133+ CRC cells. As shown in Fig. 2B, USP22 was overexpressed in CD133+ Caco2 and HCT15 stem cells. The CD133+ Caco 2 and HCT15 stem cells were then induced to differentiate. The expression of stem cell markers, including CD133, CD44 and Sox2, were found to be downregulated while differentiation marker Xbp1 was observed to be upregulated, suggesting successful induction of differentiation (Fig. $2 \mathrm{C}$ and 2D). Both USP22 mRNA and protein were also downregulated after differentiation (Fig. 2C and 2D). Furthermore, we decreased USP22 using siRNA and examined stemness and differentiation markers. As we expected, downregulation of USP22 decreased CRC cell stemness markers and increased differentiation markers (Fig. 3A and $3 \mathrm{~B})$. We then performed

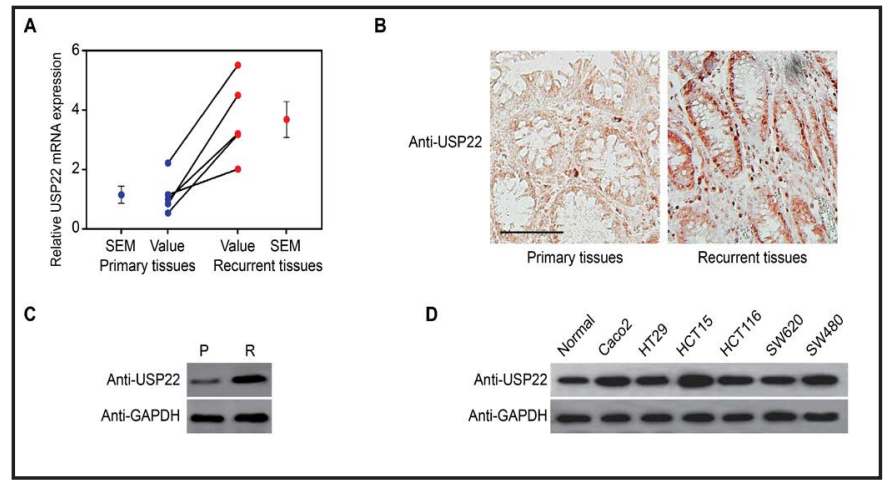

Fig. 1. USP22 expression was consistently increased in recurrent CRC tissues. (A) USP22 mRNA expression in four paired primary and recurrent CRC tissues by RT-PCR. (B, C) Representative immunohistochemistry staining and western blot of USP22 in primary and recurrent CRC tissues. Scale bar, $100 \mu \mathrm{m}$. (D) USP22 protein expression in normal CRC tissues and six CRC cell lines.

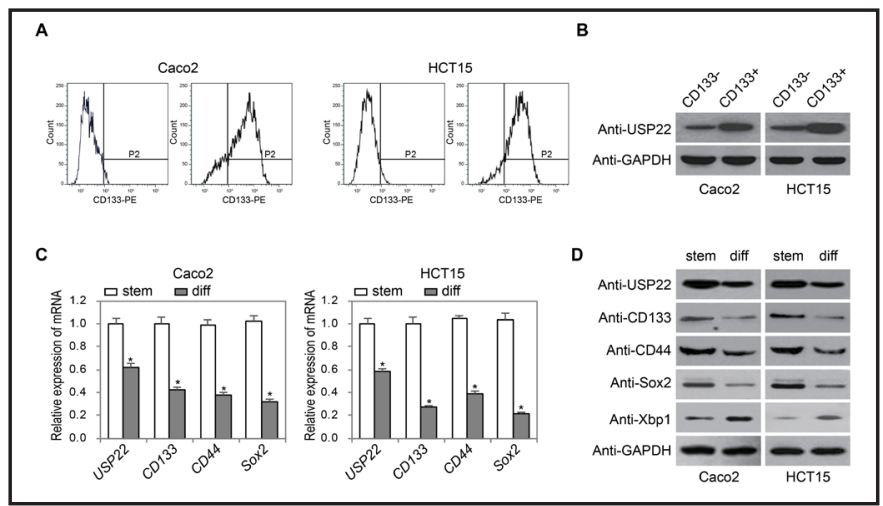

Fig. 2. USP22 is increased in CRC stem cells. (A) Caco2 and HCT15 CD133+ stem cells were isolated with CD133 MicroBeads. Flow cytometry was used to analyze the expression of CD133 before and after isolation. (B) Western blot analysis of USP22 protein in CD133- and CD133+ Caco2 and HCT15 stem cells. (C) Caco2 and HCT15 CD133+ stem cells were induced to differentiate. RTPCR analysis of USP22, CD133, CD44 and Sox2 mRNA levels were analyzed before and after differentiation. ${ }^{*} \mathrm{P}<0.05$ compared with stem cells. (D) Western blot analysis of USP22, CD133, CD44, Sox2 and Xbp1 protein levels were analyzed before and after differentiation. 


\section{Cellular Physiology Cell Physiol Biochem 2018;46:1412-1422 \begin{tabular}{l|l} 
DOI: 10.1159/000489156 & Ond Biochemistry \\
Published online: AprII23, 2018 & $\begin{array}{l}\text { 2018 The Author(s). Published by S. Karger AG, Basel } \\
\text { www.karger.com/cpb }\end{array}$
\end{tabular} \\ Jiang et al.: USP22 Contributes to Colorectal Cancer Stemness and Chemoresistance}

sphere-forming assays using USP22 knockdown cells. As shown in Fig. 3C and 3D, knockdown of USP22 reduced sphere number and single sphere size of CD133+ Caco2 and HCT15 cells. In addition, downregulation of USP22 significantly inhibited colorectal stem cell proliferation (Fig. 3E). We next performed in vivo limiting dilution assays by subcutaneously transplanting Caco 2 cells (CD133- or CD133+ with or without USP22 downregulation) into nude mice. As shown in Fig. 3F, the USP22 knockdown groups needed more cells to form subcutaneous tumors. Consistent with the results of the in vitro assays, CD133 staining in USP22 knockdown tumors was weaker than in the control group (Fig. 3G). Overall, these results indicate that USP22 expression is necessary for CRC stemness and tumorigenesis, in vitro and in vivo.

\section{Knockdown of USP22 attenuated the Wnt/ $\beta$-catenin signaling pathway}

We next explored the mechanism by which USP22 regulates CRC cell stemness and tumorigenesis. Because USP22 is functionally correlated with Wnt/ $\beta$-catenin signaling regulators and proteins Foxm 1 and GSK $\beta[7,16]$, we hypothesized that USP22 maintains CRC cell stemness and tumorigenesis through Wnt/ $\beta$-catenin signaling. To explore this hypothesis, we performed RT-PCR and western blot assays for Wnt/ $\beta$-catenin signaling target genes (Axin2, MYC and Cyclin D1) in USP22 knockdown Caco2 and HCT15 stem cells. As shown in Fig. 4A and 4B, these genes were significantly downregulated in CRC cells with USP22 knockdown. We then performed Wnt luciferase activity assays in USP22 knockdown

Fig. 3. Knockdown of USP22 reduced CRC cell stemness. (A, B) USP22 was decreased in Caco2 and HCT15 stem cells by siRNA method. The cells were subjected to RT-PCR (A) and western blot (B) for USP22, CD133, CD44, Sox2 and Xbp1 expression. (C) USP22 knockdown $\mathrm{Caco} 2$ and HCT15 stem cells were subjected to sphere formation assays. Number of spheres was quantified. ${ }^{*} \mathrm{P}<0.05$ compared with scramble cells. Scale bar, $100 \mu \mathrm{m}$. (D) Sphere formation assays of single cells were performed. Sphere diameter was quantified. ${ }^{*} \mathrm{P}<0.05$ compared with scramble cells. Scale bar, $100 \mu \mathrm{m}$. (E) MTT analysis of USP22 knockdown $\mathrm{Caco} 2$ and HCT15 stem cells. (F) In vivo limiting dilution

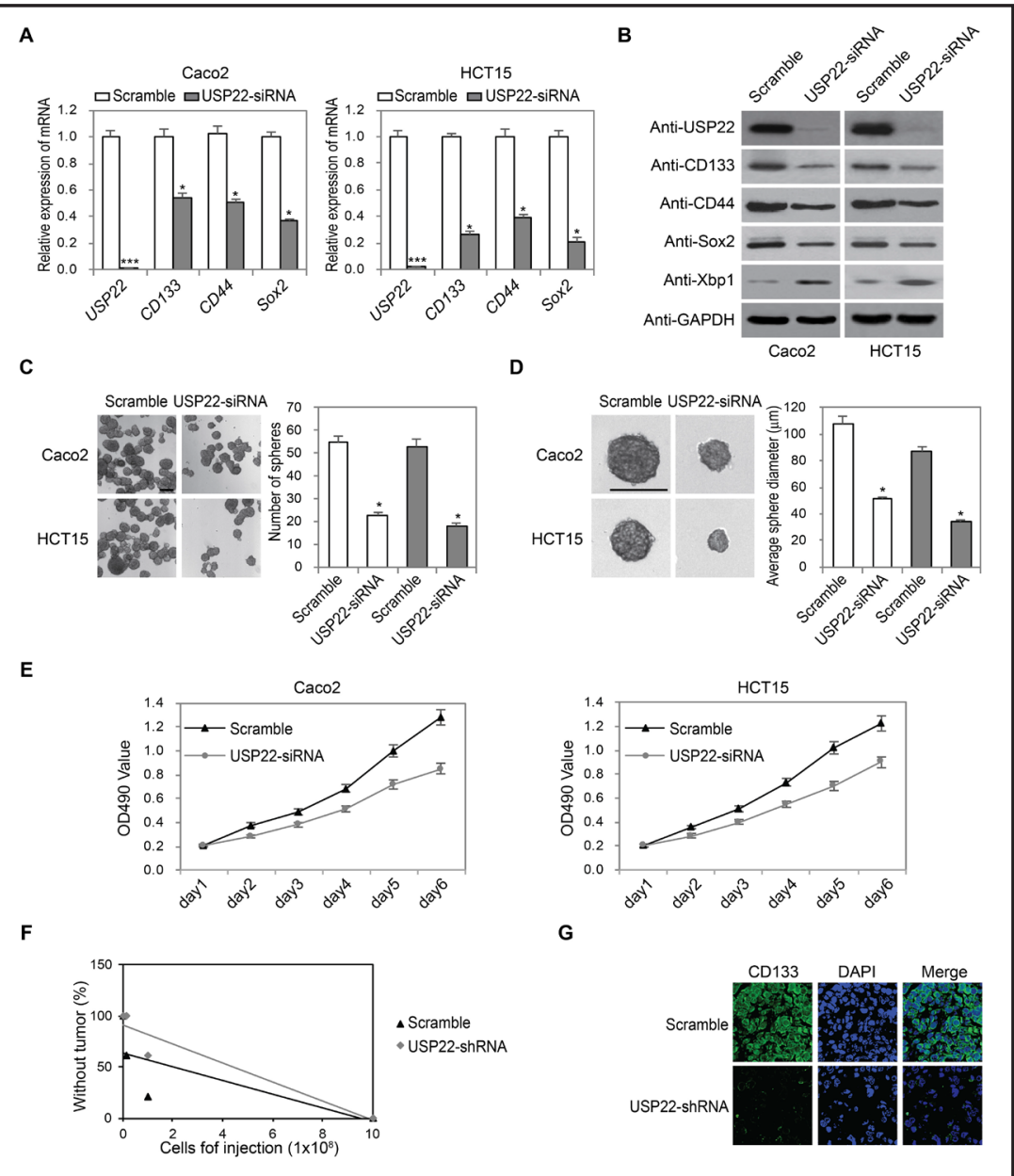
assays were performed in Caco2 cells of control and USP33 knockdown. (G) Fluorescent staining of CD133 in subcutaneous tumors derived from CRC cells with USP22 knockdown or control. 
Fig. 4. Knockdown of USP22 attenuated Wnt/ $\beta$-catenin signaling pathway. (A, B) RT-PCR (A) and western blot $(\mathrm{B})$ analysis of $\mathrm{Wnt} / \beta$ catenin signaling target gene mRNA and protein levels in USP22 knockdown CRC cells. (C) Wnt luciferase analysis was performed in USP22 knockdown Caco 2 and HCT15 stem cells. $* \mathrm{P}<0.05$ and \# $\mathrm{P}<0.05$ compared with scramble cells without or with Wnt3a treatment, respectively. (D) Western blot analysis of $\beta$-catenin protein in both whole cells (upper) and nucleus (lower) in Caco2 and HCT15 stem cells with USP22 knockdown. (E) RT-PCR analysis of Wnt/ $\beta$-catenin signaling component mRNA levels in USP22 knockdown Caco 2 and HCT15 cells. ${ }^{*} \mathrm{P}<0.05$ compared with scramble cells.

Caco2 and HCT15 stem cells with or without Wnt3a treatment. These assays showed that Wnt luciferase activity was attenuated in USP22 knockdown CRC cells (Fig. 4C). Additionally, a simultaneous decrease of total and nuclear $\beta$-catenin protein levels was observed in Caco 2 and HCT15 stem cells with USP22 knockdown (Fig. 4D). Finally, RT-PCR of Wnt/ $\beta$-catenin signaling components, including Wnt1, Wnt2b, Wnt3a, LRP5, LRP6, Axin, GSK3 $\beta$ and APC, showed strong downregulation of Wnt1, Wnt2b and Wnt3a expression (Fig. 4E). Taken together, these findings indicate that USP2 2 is required for Wnt/ $\beta$-catenin signaling pathway activity in CRC cells.

\section{Knockdown of USP22 reduced CRC cell chemoresistance}

It has been reported that tumor initiating stem cells or cancer stem cells from the original tumor are the main reasons for tumor resistance to chemotherapy [17]. Our previous results demonstrated that USP22 is required for CRC cell stemness and tumorigenesis. We next determined whether USP22 promotes CRC cell chemoresistance. To start, we generated 5-FU resistant CRC cells and examined their levels of USP22 expression. RT-PCR and western blot showed that both mRNA and protein expression of USP22 were increased in 5-FU resistant cells (Fig. 5A and 5B). We then decreased USP22 expression in 5-FU resistant Caco2 and HCT15 cells (Fig. 5C). Sphere formation and cell viability assays revealed that USP22 knockdown significantly reduced the rate of sphere formation and viability of chemoresistant CRC cells (Fig. 5D and 5E). In addition, we observed reduced cell viability in normal Caco2 and HCT15 cells under 5-FU treatment (Fig. 5F).

USP22 mediated CRC cell chemoresistance through Wnt/ $\beta$-catenin signaling

$W n t / \beta$-catenin signaling is one of the key signaling pathways involved in cancer stem cell chemoresistance $[11,12]$, and our previous data have demonstrated that USP22 regulates 
Fig. 5. Knockdown of USP22 reduced chemoresistance in colorectal cancer cells. (A, B) RT-PCR (A) and western blot (B) analysis of USP22 mRNA and protein levels in 5-FU resistant $\mathrm{Caco} 2$ and HCT15 cells. ${ }^{*} \mathrm{P}<0.05$ compared with primary cells. (C) Western blot analysis of USP22 protein levels in 5-FU resistant Caco2 and HCT15 cells after USP22 knockdown by siRNA. (D) Sphere formation assays in 5-FU resistant Caco 2 and HCT15 cells with USP22 knockdown under $30 \mu \mathrm{m} 5$-FU treatment. $\quad * P<0.05$ compared with scramble cells. (E) Cell viability assays in 5-FU resistant Caco 2 and HCT15 cells with USP22 knockdown. $* \mathrm{P}<0.05$ compared with scramble cells. (F) Cell viability

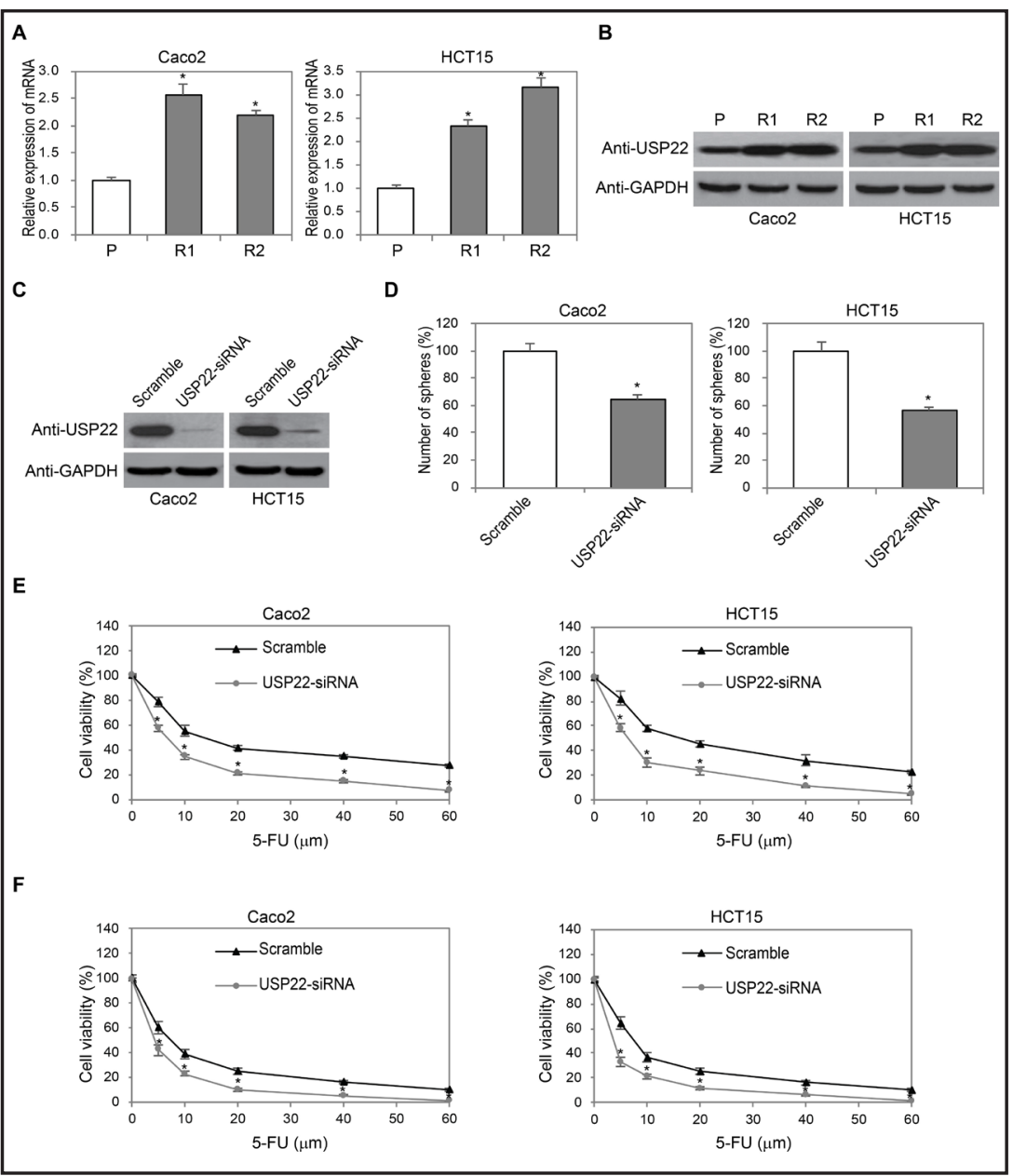
assays in normal Caco 2 and HCT15 cells with USP22 knockdown. ${ }^{*} \mathrm{P}<0.05$ compared with scramble cells.

Wnt/ $\beta$-catenin signaling. To determine whether USP22 affects CRC cell chemoresistance through Wnt/ $\beta$-catenin signaling, we knocked down USP22 in 5-FU resistant Caco 2 cells. These cells were then treated with 5 -FU, and the downstream genes of Wnt/ $\beta$-catenin signaling were examined. We found that 5 -FU significantly promoted the expression of Wnt/ $\beta$-catenin signaling target genes. However, 5-FU failed to elevate gene expression in USP22 knockdown cells (Fig. 6A and 6B). In addition, Wnt luciferase activity and $\beta$-catenin expression were not promoted by 5-FU in USP22 knockdown CRC cells (Fig. 6C and 6D). We then increased $\beta$-catenin expression in USP22 knockdown cells (Fig. 6E). These cells were subjected to cell viability assays under 5-FU treatment. The results showed that overexpression of $\beta$-catenin totally rescued cell viability inhibited by knockdown of USP22 (Fig. 6F).

\section{Discussion}

In this study, we have identified a critical role for USP22 in the development of CRC stemness and chemoresistance. Our mechanistic findings establish that USP22 does so through the Wnt/ $\beta$-catenin signaling pathway. Ultimately, this study opens the possibility of targeting USP22 expression to increase CRC susceptibility to 5-FU in a clinical setting.

USP22 is a deubiquitinating enzyme and putative CRC marker that has been shown to promote the pathological processes of various malignancies, including tumor recurrence 
Fig. 6. USP22 reduces 5-FU induced chemoresistance through Wnt/ $\beta$-catenin signaling pathway. (A, B) RT-PCR (A) and western blot (B) analysis of $\mathrm{Wnt} / \beta$ catenin signaling target gene mRNA levels in USP22 knockdown Caco 2 cells treated with or without 5 -FU. ${ }^{*} \mathrm{P}<0.05$ compared with scramble cells. (C) Wnt luciferase analysis was performed in USP22 knockdown Caco 2 cells treated with or without 5-FU. ${ }^{*} \mathrm{P}<0.05$ compared with scramble cells. (D) Western blot analysis of $\beta$-catenin levels in USP22 knockdown

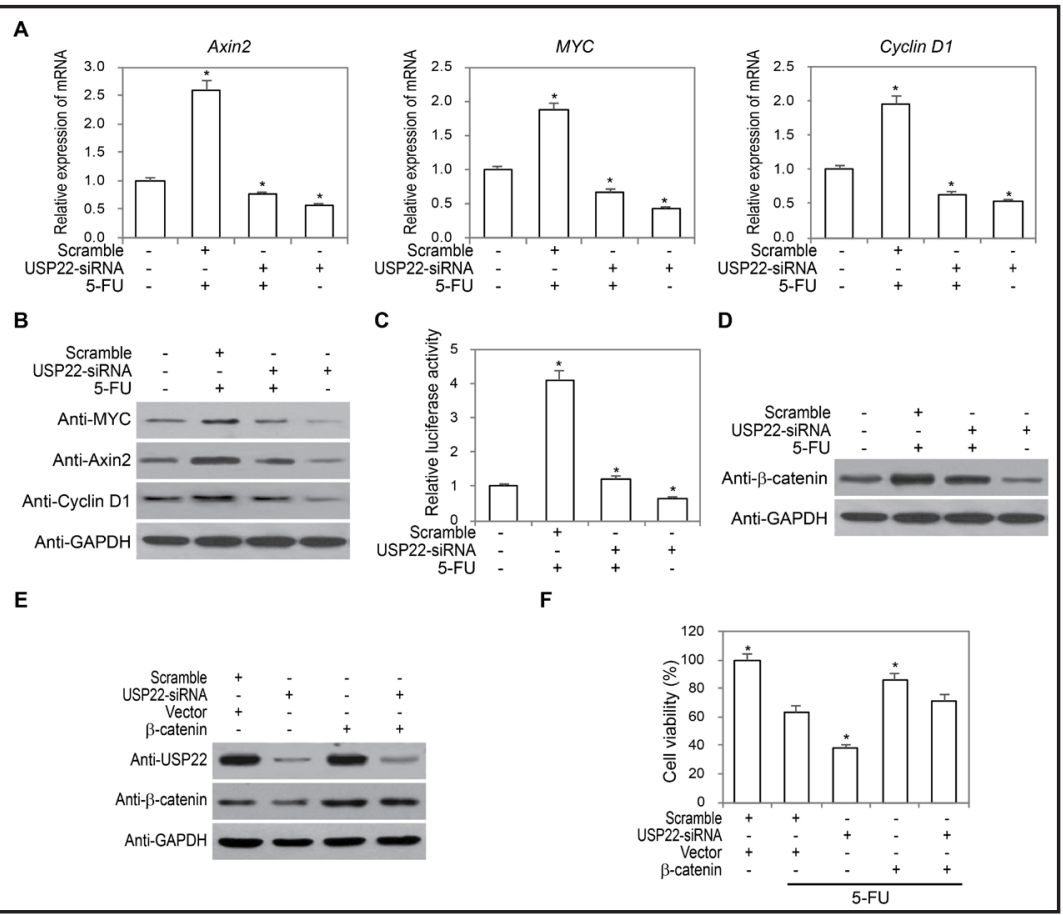
Caco 2 cells treated with or without 5-FU. (E) Expression of USP22 and $\beta$-catenin in USP22 knockdown Caco 2 cells with or without $\beta$-catenin overexpression. (F) Cell viability assays in USP22 knockdown Caco 2 cells with or without $\beta$-catenin overexpression under 5 -FU treatment. ${ }^{*} \mathrm{P}<0.05$ compared with control cells under 5 -FU treatment.

and metastasis, [18-21]. In its most basic functions, USP22 promotes the G1/S cell cycle transition and cell proliferation [7-9]. It has been shown to upregulate FoxM1 expression via $\beta$-catenin nuclear localization and to regulate the SIRT-STAT2 signaling pathway [7, 22]. Despite evidence that USP22 causes poor CRC prognoses, prior to this study, it was unclear how it does so. The results of this study reveal that in CRC, USP22 acts through the Wnt/ $\beta$ catenin signaling pathway to maintain CRC cell stemness and cause chemoresistance.

CRC recurrence occurs for a variety of reasons, including CSC reservoirs that haven't been resected or eliminated by chemotherapy, as well as molecular pathways such as Wnt and Notch [23]. To determine whether USP22 plays a role in CRC recurrence, we analyzed USP22 expression levels in paired fresh primary and recurrent CRC tissues from four human patients and found that, in fact, USP22 was overexpressed in recurrent CRC as compared to primary CRC. After determining that USP22 was upregulated in putative CSCs (CD133+ CRC cells) and required for CRC stemness and tumorigenesis, it seemed likely that USP22 overexpression is in fact crucial to CRC recurrence. Because USP22 is functionally related to Wnt signaling and Wnt signaling overactivation is a hallmark of CRC, it seemed likely that USP22 exerted its effects on CRC stemness through the Wnt/ $\beta$-catenin pathway $[7,24]$.

The canonical Wnt pathway is an evolutionarily conserved mechanism of stem cell regulation [24, 25]. Aberrant regulation of the Wnt pathway causes malignant proliferation, and its activation helps maintain the CSC reservoir that contributes to CRC stemness, recurrence, and chemoresistance [26, 27]. While there are many regulators of the Wnt pathway, none have been identified that account for these properties of CRC. Using USP22 knockdown CRC cell lines, we demonstrated that USP22 activity is necessary for the Wnt/ $\beta$ catenin pathway.

Having established the necessity of USP22 for CRC stemness, tumorigenesis, and the Wnt/ $\beta$-catenin pathway, we needed to determine whether USP22 is responsible for CRC chemoresistance and, if so, to reveal its mechanism of action. CRC chemoresistance is the 


\section{Cellular Physiology Cell Physiol Biochem 2018;46:1412-1422 \begin{tabular}{l|l} 
and Biochemistry Published onlıne: April 23, 2018 & $\begin{array}{l}\text { (c) } 2018 \text { The Author(s). Published by S. Karger AG, Basel } \\
\text { www.karger.com/cpb }\end{array}$
\end{tabular}}

Jiang et al.: USP22 Contributes to Colorectal Cancer Stemness and Chemoresistance

main reason why the disease's prognosis is so poor [28]. Prior to this study, it had been shown that chemoresistance can develop due to the maintenance of CSC reservoirs and the upregulation of signaling pathways such as Wnt $[26,27,29]$. We therefore hypothesized that USP2 2 was required for CRC chemoresistance and that it mediated chemoresistance through Wnt/ $\beta$-catenin signaling. Indeed, USP22 expression was necessary for chemoresistance in 5 -FU resistant CRC cell lines, and decreasing USP22 expression reduced chemoresistance. Finally, 5-FU did not elevate downstream genes of Wnt/ $\beta$-catenin signaling in USP22 knockdown cells, indicating that USP22 is necessary for chemoresistance mediated through Wnt/ $\beta$-catenin signaling.

\section{Conclusion}

These results demonstrate that USP22 plays a crucial role in CRC stemness, recurrence, tumorigenesis, and chemoresistance--largely through its functional relationship to the Wnt/ $\beta$-catenin signaling pathway. While future studies are necessary to determine the mechanism of USP22 overexpression, the present study provides compelling evidence for targeting USP22 in the treatment of CRC.

\section{Acknowledgements}

This study was supported by the Science and Technology Research Project of Heilongjiang Province Education Department (No. 12541460).

\section{Disclosure Statement}

All authors declared no conflicts of interest in this work.

\section{References}

1 Marin JJ, Sanchez de Medina F, Castano B, Bujanda L, Romero MR, Martinez-Augustin O, Moral-Avila RD, Briz 0: Chemoprevention, chemotherapy, and chemoresistance in colorectal cancer. Drug Metab Rev 2012;44:148-172.

2 Garza-Trevino EN, Said-Fernandez SL, Martinez-Rodriguez HG: Understanding the colon cancer stem cells and perspectives on treatment. Cancer Cell Int 2015;15:2.

3 Yoshimatsu K, Ishibashi K, Hashimoto M, Umehara A, Yokomizo H, Yoshida K, Fujimoto T, Iwasaki K, Ogawa K: [Effect of cimetidine with chemotherapy on stage IV colorectal cancer]. Gan To Kagaku Ryoho 2003;30:1794-1797.

-4 O'Brien CA, Pollett A, Gallinger S, Dick JE: A human colon cancer cell capable of initiating tumour growth in immunodeficient mice. Nature 2007;445:106-110.

5 Dave B, Mittal V, Tan NM, Chang JC: Epithelial-mesenchymal transition, cancer stem cells and treatment resistance. Breast Cancer Res 2012;14:202.

6 Gupta PB, Chaffer CL, Weinberg RA: Cancer stem cells: mirage or reality? Nat Med 2009;15:1010-1012.

7 Ning Z, Wang A, Liang J, Xie Y, Liu J, Feng L, Yan Q Wang Z: USP22 promotes the G1/S phase transition by upregulating FoxM1 expression via beta-catenin nuclear localization and is associated with poor prognosis in stage II pancreatic ductal adenocarcinoma. Int J Oncol 2014;45:1594-1608.

-8 Jamieson C, Sharma M, Henderson BR: Targeting the beta-catenin nuclear transport pathway in cancer. Semin Cancer Biol 2014;27:20-29.

-9 Zhang XY, Varthi M, Sykes SM, Phillips C, Warzecha C, Zhu W, Wyce A, Thorne AW, Berger SL, McMahon SB: The putative cancer stem cell marker USP22 is a subunit of the human SAGA complex required for activated transcription and cell-cycle progression. Mol Cell 2008;29:102-111. 


\section{Cellular Physiology Cell Physiol Biochem 2018;46:1412-1422 \begin{tabular}{l|l} 
DOI: 10.1159/000489156 & and Biochemistry 2018 The Author(s). Published by S. Karger AG, Basel \\
wuww.karger.com/cpb
\end{tabular}

10 Liu YL, Yang YM, Xu H, Dong XS: Increased expression of ubiquitin-specific protease 22 can promote cancer progression and predict therapy failure in human colorectal cancer. J Gastroenterol Hepatol 2010;25:18001805.

11 Mohammed MK, Shao C, Wang J, Wei Q, Wang X, Collier Z, Tang S, Liu H, Zhang F, Huang J, Guo D, Lu M, Liu F, Liu J, Ma C, Shi LL, Athiviraham A, He TC, Lee MJ: Wnt/beta-catenin signaling plays an ever-expanding role in stem cell self-renewal, tumorigenesis and cancer chemoresistance. Genes Dis 2016;3:11-40.

-12 Johnsen JI, Wickstrom M, Baryawno N: Wingless/beta-catenin signaling as a modulator of chemoresistance in cancer. Mol Cell Oncol 2016;3:e1131356.

13 Vermeulen L, Snippert HJ: Stem cell dynamics in homeostasis and cancer of the intestine. Nat Rev Cancer 2014;14:468-480.

14 Fearon ER: Molecular genetics of colorectal cancer. Annu Rev Pathol 2011;6:479-507.

15 Chen JF, Luo X, Xiang LS, Li HT, Zha L, Li N, He JM, Xie GF, Xie X, Liang HJ: EZH2 promotes colorectal cancer stem-like cell expansion by activating p21cip1-Wnt/beta-catenin signaling. Oncotarget 2016;7:4154041558.

16 Zhou A, Lin K, Zhang S, Chen Y, Zhang N, Xue J, Wang Z, Aldape KD, Xie K, Woodgett JR, Huang S: Nuclear GSK3beta promotes tumorigenesis by phosphorylating KDM1A and inducing its deubiquitylation by USP22. Nat Cell Biol 2016;18:954-966.

17 Paldino E, Tesori V, Casalbore P, Gasbarrini A, Puglisi MA: Tumor initiating cells and chemoresistance: which is the best strategy to target colon cancer stem cells? Biomed Res Int 2014;2014:859871.

18 Liu YL, Yang YM, Xu H, Dong XS: Aberrant expression of USP22 is associated with liver metastasis and poor prognosis of colorectal cancer. J Surg Oncol 2011;103:283-289.

19 Lin Z, Tan C, Qiu Q Kong S, Yang H, Zhao F, Liu Z, Li J, Kong Q, Gao B, Barrett T, Yang GY, Zhang J, Fang D: Ubiquitin-specific protease 22 is a deubiquitinase of CCNB1. Cell Discov 2015;1:

-20 Glinsky GV, Berezovska O, Glinskii AB: Microarray analysis identifies a death-from-cancer signature predicting therapy failure in patients with multiple types of cancer. J Clin Invest 2005;115:1503-1521.

-21 Glinsky GV: Genomic models of metastatic cancer: functional analysis of death-from-cancer signature genes reveals aneuploid, anoikis-resistant, metastasis-enabling phenotype with altered cell cycle control and activated Polycomb Group (PcG) protein chromatin silencing pathway. Cell Cycle 2006;5:1208-1216.

22 Ao N, Liu Y, Feng H, Bian X, Li Z, Gu B, Zhao X: Ubiquitin-specific peptidase USP22 negatively regulates the STAT signaling pathway by deubiquitinating SIRT1. Cell Physiol Biochem 2014;33:1863-1875.

23 Vaiopoulos AG, Kostakis ID, Koutsilieris M, Papavassiliou AG: Colorectal cancer stem cells. Stem Cells 2012;30:363-371.

24 de Sousa EMF, Vermeulen L: Wnt Signaling in Cancer Stem Cell Biology. Cancers (Basel) 2016;8:

25 Basu S, Haase G, Ben-Ze'ev A: Wnt signaling in cancer stem cells and colon cancer metastasis. F1000Res 2016;5:

26 Reya T, Clevers H: Wnt signalling in stem cells and cancer. Nature 2005;434:843-850.

-27 Ordonez-Moran P, Dafflon C, Imajo M, Nishida E, Huelsken J: HOXA5 Counteracts Stem Cell Traits by Inhibiting Wnt Signaling in Colorectal Cancer. Cancer Cell 2015;28:815-829.

28 Hammond WA, Swaika A, Mody K: Pharmacologic resistance in colorectal cancer: a review. Ther Adv Med Oncol 2016;8:57-84.

29 Han P, Li JW, Zhang BM, Lv JC, Li YM, Gu XY, Yu ZW, Jia YH, Bai XF, Li L, Liu YL, Cui BB: The lncRNA CRNDE promotes colorectal cancer cell proliferation and chemoresistance via miR-181a-5p-mediated regulation of Wnt/beta-catenin signaling. Mol Cancer 2017;16:9. 